\title{
Presencia de Holochilus chacarius Thomas, 1906 (Mammalia, Rodentia, Sigmodontinae) en el Delta Medio del río Paraná, Entre Ríos, Argentina
}

\author{
Paula COURTALON ${ }^{1}$, Gastón LO COCO ${ }^{1,2}$ \& Roberto F. BÓ1,3
}

\begin{abstract}
${ }^{1}$ Grupo de Investigaciones en Ecología de Humedales. Departamento de Ecología, Genética y Evolución, Facultad de Ciencias Exactas y Naturales, Universidad de Buenos Aires. Ciudad Universitaria, Pabellón II, 4to Piso, 1428 Ciudad Autónoma de Buenos Aires, Argentina. pcourtalon@gmail.com, ${ }^{2}$ gastonlococo@gmail.com, ${ }^{3}$ rober@ege.fcen.uba.ar
\end{abstract}

\begin{abstract}
Holochilus chacarius Thomas, 1906 (Mammalia, Rodentia, Sigmodontinae) in Middle Delta of the Paraná River, Entre Ríos, Argentina. We describe the presence of Holochilus chacarius in the Victoria Island Zone included in the Middle Delta of the Paraná River (Entre Ríos, Argentina). We collected and analysed pellets of the Striped Owl (Pseudoscops clamator). All cranio dental items that were identified and measured belong to the Chaco Marsh Rat. We postulate that its occurrence is becoming less casual and is associated with the high climate variability and extreme events of flooding and drought that have affected the area in recent years. These events would favour the ingression and eventual establishment of these rodents in the study area.
\end{abstract}

Key words: Holochilus chacarius, Sigmodontinae, Middle Delta of the Paraná River, Entre Ríos, Argentina.

Resumen: Se describe la presencia de Holochilus chacarius en la Zona de Islas del Departamento Victoria, ubicada en el Delta Medio del río Paraná (Entre Ríos, Argentina). Se recolectaron y analizaron egagrópilas de lechuzón orejudo (Pseudoscops clamator). Todos los restos cráneo-dentarios identificados y medidos pertenecen a la rata nutria chaqueña. Se postula que su aparición es cada vez menos ocasional y se encuentra asociada con la elevada variabilidad climática y los eventos extremos de inundación y sequía que afectaron el área en los últimos años. Estos eventos favorecerían el ingreso y la eventual instalación de estos roedores en el área.

Palabras clave: Holochilus chacarius, Sigmodontinae, Delta Medio del río Paraná, Entre Ríos, Argentina.

\section{INTRODUCCIÓN}

La rata nutria chica o rata nutria chaqueña (Holochilus chacarius) es un roedor sigmodontino, cuya ecología es relativamente poco conocida tanto en Argentina como en el resto de Latinoamérica. $\mathrm{Al}$ igual que otros representantes del género Holochilus, tiene hábitos semiacuáticos y herbívoros (Hershkovitz, 1955; Parera, 2002). Construye nidos con distintos tipos de herbáceas y ramas de leñosas y eventualmente cuevas acondicionadas con materiales similares (Massoia, 1976; Udrizar Sauthier et al., 2010).

Hasta hace pocos años atrás, se consideraba que $H$. chacarius era típica del Chaco Húmedo, distribuyéndose desde el Paraguay hasta el centro-norte de las provincias de Santa Fe y Entre Ríos, siendo su límite sur los $\mathrm{S} 31^{\circ} 38^{\prime}-\mathrm{O} 60^{\circ}$ 42', sobre la margen occidental del río Paraná
(Massoia, 1971; Massoia, 1976). Sin embargo, de un estudio realizado por Voglino et al. (2004) surge que esta especie se halla presente bastante más al sur, alcanzando los $\mathrm{S} 33^{\circ} 35^{\prime}$ y $\mathrm{O}$ $59^{\circ}$ 49', en la Cueva La Salamanca, San Pedro, provincia de Buenos Aires. En la Zona de Islas del Departamento Victoria (ZIDV, Entre Ríos), donde también fue descripta la presencia de esta especie por los autores anteriormente señalados, las ratas del género Holochilus se encuentran normalmente en los bajos y medias lomas de las islas compartiendo su hábitat con el coipo o falsa nutria (Myocastor coypus) (Bó, en prensa).

La presencia del género Holochilus ha sido descripta en Colombia por Valencia (1988) determinando la presencia de $H$. brasiliensis en los departamentos de Tolima, Cesar, Sucre y las zonas arroceras de los llanos orientales de Colombia. En Venezuela, Agüero (1986) reportó la presencia de 




Fig. 1. La Zona de Islas del Departamento Victoria (ZIDV) en el Delta Medio del Río Paraná (Entre Ríos, Argentina). $\mathbf{\Delta}$ : Área de muestreo.

$H$. venezuelae en cultivos de arroz en los estados Portuguesa, Guárico y Barinas y en cultivos de caña de azúcar del estado Portuguesa (Agüero et al., 1985).

A pesar de ello es muy poco lo que se conoce sobre la biología y la ecología del género en Argentina y en el resto de Latinoamérica.

En este contexto, el objetivo de este trabajo fue documentar la presencia de $H$. chacarius y discutir sus posibles causas en la Zona de Islas del Departamento Victoria (ZIDV), Entre Ríos, Argentina.

\section{MATERIALES Y MÉTODOS}

El estudio se llevó a cabo durante la primavera de 2008 en la ZIDV, ubicada en el Delta Medio del Río Paraná (Malvárez et al., 1999) (Fig. 1). La misma se encuentra influenciada, fundamentalmente, por el régimen del río Paraná, presentando un período de creciente o de "aguas altas" (desde marzo a julio) y uno de estiaje o de "aguas bajas" (desde agosto a febrero) con leves repuntes en octubre y noviembre. Ocasionalmente (aunque en forma relativamente más frecuente en los últimos años), se producen eventos de características extremas: inundaciones y/o sequías, que afectan la fisonomía, composición y diversidad de la subregión y las actividades humanas predominantes (Malvárez et al., 1999; Bó \& Malvárez, 1999; Bó et al., 2008). En el verano del año 2007, en el área de estudio tuvo lugar un evento de inundación extrema que alcanzó su pico máximo en el otoño. Posteriormente, los niveles del agua bajaron en forma relativamente rápida y drástica, generando un período de sequía extremo que se extendió desde la primavera de 2007 hasta fines de 2008.

Los distintos ambientes de humedal que constituyen el patrón de paisaje característico, al ubicarse en diferentes posiciones topográficas relativas (altos, medias lomas altas, medias lomas bajas y bajos), se encuentran sometidos a distintas condiciones de anegamiento o inundación (desde temporarias a permanentes) y tienen niveles de agua variables a lo largo del año. Esto determina no sólo diferentes fisonomías vegetales (praderas de herbáceas de distinto tipo y porte en medias lomas y bajos y bosques monoespecíficos en los escasos altos presentes) sino también una alta diversidad biótica y una elevada variabilidad de la misma tanto en el espacio como en el tiempo. En el caso de la fauna silvestre, esta última se distingue por las presencia de numerosas especies particularmente adaptadas a la dinámica hidrológica mencionada, tales como los roedores del género Holochilus (Bó \& Malvárez, 1999; Malvárez \& Bó, 2000).

Se recolectaron y analizaron egagrópilas de lechuzón orejudo (Pseudoscops clamator) en un posadero que utilizaba una pareja de dicha especie. El mismo se encontraba en un bosque abierto de sauce criollo (Salix humboldtiana) ubicado en la porción SE del arroyo Barrancoso (S $32^{\circ}$ $57^{\prime} 21.7^{\prime \prime}$ - O $\left.60^{\circ} 02^{\prime} 57^{\prime \prime}\right)$. Las egagrópilas fueron pesadas en balanza digital (Ohaus CS 200, precisión de $0,01 \mathrm{~g}$ ), medidas con regla y disgregadas manualmente bajo campana (Simmons \& Muñoz-Saba, 2005).

Todas las medidas cráneo-dentarias se tomaron con calibre KLM (Shangay, China, escala 150 $\mathrm{mm}$, New Jersey, USA, precisión de 0,02 mm). La determinación taxonómica de los restos cráneodentarios fue realizada siguiendo los criterios de identificación y medición propuestos por Voglino et al. (2004) basados en el análisis de la morfología y dimensiones de las hemimandíbulas y la serie dentaria. 

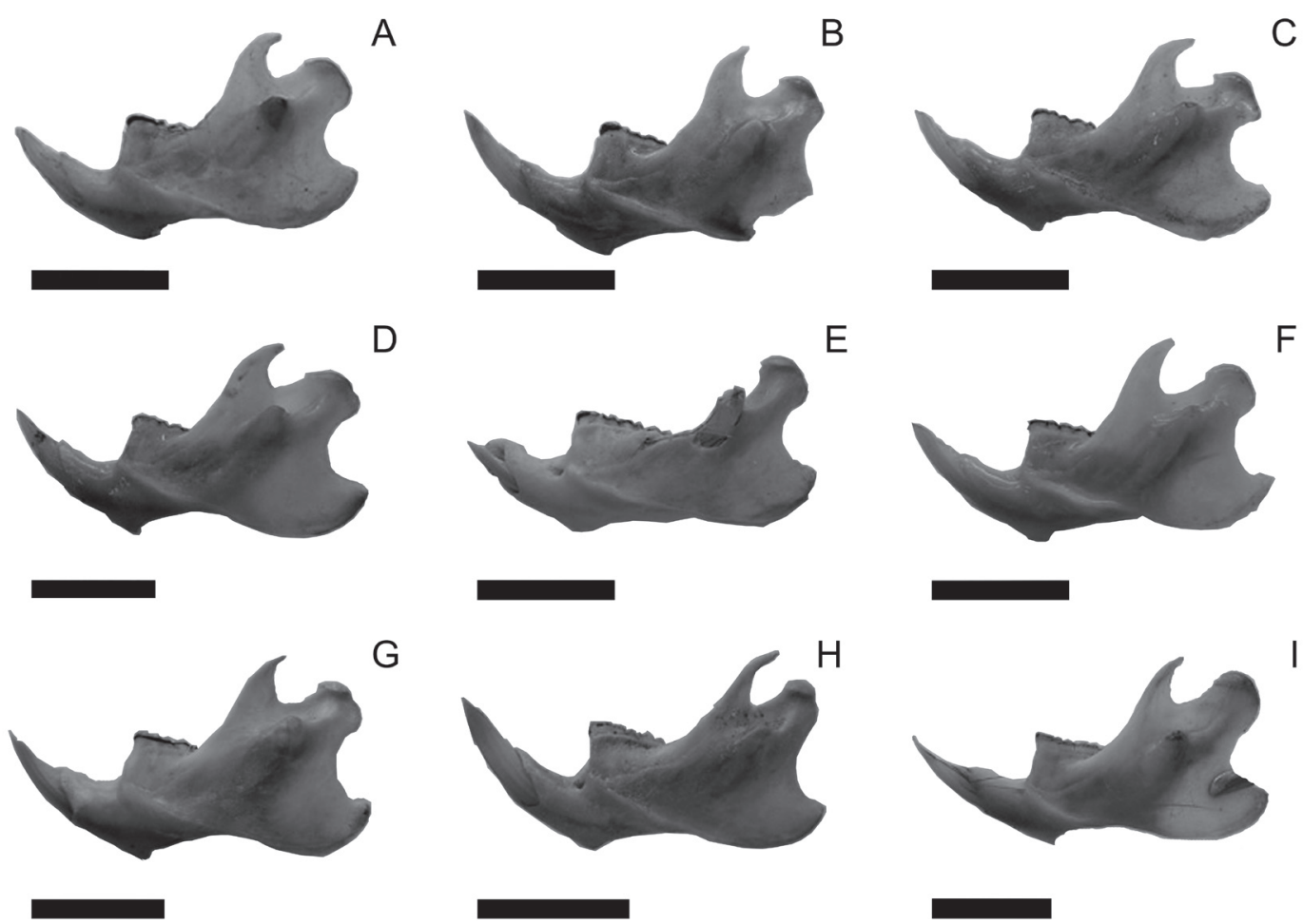

Fig. 2. Hemimandíbulas izquierdas en vista labial de los individuos de H. chacarius (A-I) hallados en las egagrópilas de Pseudoscops clamator recolectadas en la Zona de Islas del Departamento Victoria (Entre Ríos, Argentina). Barra de escala: $1 \mathrm{~cm}$.


Fig. 3. Series molares inferiores izquierdas en vista oclusal de los individuos de H. chacarius (A-I) hallados en las egagrópilas de Pseudoscops clamator recolectadas en la Zona de Islas del Departamento Victoria (Entre Ríos, Argentina). Barra de escala: $1 \mathrm{~mm}$

\section{RESULTADOS}

En cada una de las egagrópilas analizadas ( $\mathrm{N}$ = 18) se registró sólo un ítem presa. La totalidad de los restos óseos pertenecieron al orden Rodentia y todos los cráneos y hemimandíbulas hallados $(\mathrm{N}=9)$ correspondieron a la Rata
Nutria Chaqueña (Holochilus chacarius).

En promedio, los cráneos midieron 37,38 \pm $2,33 \mathrm{~mm}$ ( $\pm \mathrm{DS})$, las hemimandíbulas inferiores izquierdas midieron $26,78 \pm 1,56 \mathrm{~mm}( \pm \mathrm{DS}) \mathrm{y}$ las series molares inferiores izquierdas midieron $7,59 \pm 0,30 \mathrm{~mm}( \pm \mathrm{DS})($ Tabla 1$)$.

La morfología y longitudes de los parámetros 
Tabla 1. Medidas (mm) de la longitud del cráneo (LC), la longitud de la hemimandíbula inferior izquierda (LHi) y la longitud de la serie molar izquierda inferior (SMIi), valores medios $(\chi)$ y desviación estándar (DS) de los ejemplares de $H$. chacarius hallados en las egagrópilas de Pseudoscops clamator recolectadas en la Zona de Islas del Departamento Victoria (Entre Ríos, Argentina).

\begin{tabular}{llll}
\hline Ejemplar & LC & LHi & SMIi \\
\hline $\mathbf{1}$ & 35 & 25 & 7,3 \\
$\mathbf{2}$ & 35 & 28 & 7,5 \\
$\mathbf{3}$ & 37 & 27 & 7,1 \\
$\mathbf{4}$ & 41 & 29 & 7,8 \\
$\mathbf{5}$ & 36 & 25 & 7,7 \\
$\mathbf{6}$ & 39 & 26 & 7,8 \\
$\mathbf{7}$ & 36 & 28 & 7,5 \\
$\mathbf{8}$ & - & 25 & 8,1 \\
$\mathbf{9}$ & 40 & 28 & 7,5 \\
\hline $\boldsymbol{\chi}$ & 37,38 & 26,78 & 7,59 \\
\hline $\mathbf{D S}$ & 2,33 & 1,56 & 0,30 \\
\hline
\end{tabular}

anteriormente señalados permitieron determinar que todos los restos óseos analizados correspondían a la rata nutria chaqueña Holochilus chacarius (Figs. 2 y 3 ).

\section{DISCUSIÓN Y CONCLUSIONES}

La información obtenida en la muestra analizada coincide con la de otros trabajos, donde el género Holochilus ya había sido descripto como presa de P. clamator (Martínez et al., 1996; Pautasso 2006) incluyendo un trabajo reciente de Lo Coco et al. (2012) que señala particularmente este hecho para la ZIDV. Si bien Voglino et al. (2004) habían reportado la presencia de esta especie para la zona del Delta entrerriano, incluyendo sectores cercanos a nuestra área de estudio y para el Norte de la Provincia de Buenos Aires, se postula que la aparición de $H$. chacarius en la ZIDV varios años después de dicho hallazgo y con un $100 \%$ de dominancia en la muestra analizada, indicaría que la misma no sería ocasional. Este hecho, podría deberse, como sugieren Voglino et al. (2004), a la ausencia previa de muestreos, probables inconsistencias en los métodos de búsqueda y problemas en la identificación de la especie. Sin embargo, consideramos que también se encontraría asociado con la elevada variabilidad climática y, fundamentalmente, con los eventos extremos de sequía e inundación que afectaron la zona en los últimos años y que favorecerían el ingreso y la eventual instalación de estos roedores en el área (Bó \& Malvárez, 1999; Bó et al., 2008; Courtalon \& Bó, 2012). En períodos de inundación, los mismos llegarían desde aguas arriba, probablemente transportados por embalsados de camalote (Eichhornia crassipes) y otras especies vegetales (Quintana et al., 2002; Giraudo \& Arzamendia, 2004). Los períodos relativamente secos posteriores, favorecerían su instalación en la ZIDV, dado que contaría con suficiente recursos para satisfacer sus requerimientos de alimento y refugio, tanto en los sectores de bajo como en los de media loma y alto. Esto último le permitiría mantener poblaciones mínimas viables, incrementando, de esta manera, la diversidad de roedores sigmodontinos presentes en los humedales del Delta Medio.

El presente estudio es una contribución preliminar que aporta al conocimiento de la estructura poblacional y el uso de hábitat de Holochilus chacarius (y del género Holochilus en general) en la ZIDV. El mismo pretende contribuir también a interpretar adecuadamente sus fluctuaciones espaciales y temporales, incluyendo los probables factores ecológicos determinantes de las explosiones demográficas de este género (ratadas) tales como la ocurrida en la ZIDV durante la inundación extrema de abril de 2011 (Courtalon \& Bó, 2012).

\section{AGRADECIMIENTOS}

A Carlos Hevia y familia por su hospitalidad y apoyo durante las tareas de campo. Este trabajo fue realizado en el marco del "Proyecto Nutria. Estudios ecológicos básicos para el manejo sustentable de Myocastor coypus en Argentina". El mismo es parte de un convenio de cooperación entre la Dirección de Fauna Silvestre de la Secretaría de Ambiente y Desarrollo Sustentable de la Nación (institución financiadora) y el Grupo de Investigaciones en Ecología de Humedales del Departamento de Ecología, Genética y Evolución de la Facultad de Ciencias Exactas y Naturales de la Universidad de Buenos Aires.

\section{BIBLIOGRAFÍA}

Agüero, D. 1986. Manejo Integrado en el control de vertebrados. En: Manejo integrado de plagas. Estación Experimental Lara. Fondo Nacional de Investigaciones.

Agüero, D., J. Quevedo, I. Tovar, A. Flores, V. Martínez \& G. Espinosa. 1985. Estimación de daños y observaciones sobre la rata arrocera (Holochilus venezuelae) en caña de azúcar. Caña de Azúcar 
Agropecuarias. Barquisimeto, 3(2): 63-70. Estado Lara.

Bó, R.F. (en prensa). Modelo de aptitud de hábitat: Coipo. En: M.E. Zaccagnini, J. Bernardos \& V. Rosatti (Eds.). Serie: Modelos de aptitud de hábitat de biodiversidad de Argentina. Instituto Nacional de Tecnología Agropecuaria, INTA, Buenos Aires. $30 \mathrm{pp}$.

Bó, R.F. \& A.I. Malvárez. 1999. Las inundaciones y la biodiversidad en humedales. Un análisis del efecto de eventos extremos sobre la fauna silvestre. En: A.I. Malvárez (ed.), Tópicos sobre humedales subtropicales y templados de Sudamérica, pp. 147-168, Oficina Regional de Ciencia y Tecnología de la UNESCO para America Latina y el Caribe, Montevideo.

Bó, R.F., P. Courtalon, F. Spina, R. Fernández \& G. Porini. 2008. Los eventos extremos de sequía e inundación y sus consecuencias sobre el coipo o nutria (Myocastor coypus Molina, 1782) y la actividad de caza en el Delta Medio del Río Paraná (Argentina). En: A.V. Volpedo \& L.F. Reyes (Eds.), Efecto de cambios globales sobre los humedales de Iberoamérica, pp. 167-192, RED CYTED. Programa Iberoamericano de Ciencia y Tecnología para el Desarrollo, Buenos Aires.

Courtalon, P. \& R.F. Bó. 2012. La invasión de roedores en la autovía Rosario - Victoria (Argentina) durante el evento de inundación de otoño 2011. En: II Congreso Latinoamericano de Mastozoología. Museo argentino de Ciencias Naturales 6 al 9 de noviembre de 2012.

Giraudo, A.R \& V. Arzamendia. 2004. ¿Son los humedales fluviales de la Cuenca del Plata corredores de la biodiversidad? Los amniotas como ejemplo. En: J.J. Neiff (Ed.), Humedales de Iberoamérica. CYTED, Programa Iberoamericano de Ciencia y Tecnologia para el Desarrollo y Red Iberoamericana de Humedales (RIHU), La Habana, Cuba. Pp: 157170.

Hershkovitz, P. 1955. South American marsh rats genus Holochilus, with a summary of sigmodont rodents. Feldiana, Zoology 37: 639-673

Lo Coco, G.E., P. Courtalon \& R.F. Bó. 2012. Análisis de egagrópilas del lechuzón orejudo (Pseudoscops clamator) en la Zona de Islas de Victoria, Entre Ríos, Argentina. Nuestras Aves 57: 19-21,

Malvárez, A.I. \& R.F. Bó. 2000. Identificación de indicadores ecológicos para la detección de condiciones hidrológicas en sistemas de humedales. Cuaternario y Ciencias Ambientales 1: 37-43.

Malvárez, A.I., M. Boivín \& A. Rosato. 1999. Biodiversidad, uso de los recursos naturales y cambios en las islas del Delta Medio del Río Paraná
(Dto. Victoria, provincia de Entre Ríos, Argentina). En: S. Matteucci, O. Solbrig, J. Morello \& G. Halffter (Eds.), Biodiversidad y usos de la tierra. Conceptos y ejemplos de Latinoamérica, pp. 257290, EUDEBA, Buenos Aires.

Martinez, M.M., J.P. Isaach \& F. Donatti. 1996. Aspectos de la distribución y biología reproductiva de Asio clamator en la Provincia de Buenos Aires, Argentina. Ornitología Neotropical 7: 157-161.

Massoia, E. 1971. Caracteres y rasgos bioecológicos de Holochilus brasiliensis chacarius Thomas ("rata nutria") de la provincia de Formosa y comparaciones con Holochilus brasiliensis vulpinus (Brants) (Mammalia, Rodentia, Cricetidae). Rev. de Investigaciones Agropecuarias, INTA, Serie 1, Biología y Producción Animal 8: 13-40.

Massoia, E. 1976. Mammalia. En: R. Ringuelet (ed.), Fauna de agua dulce de la República Argentina, 44: 1-128, Fundación Editorial Ciencia y Cultura, Buenos Aires.

Parera, A. 2002. Los mamíferos de la Argentina y la región austral de Sudamérica. El Ateneo, Buenos Aires.

Pautasso, A.A. 2006. Dieta del Lechuzón Orejudo (Asio clamator) en el centro y este de la provincia de Santa Fe, Argentina. Ornitología Neotropical 17: 289-293.

Quintana, R.D, R.F. Bó \& F. Kalesnik. 2002. La vegetación y la fauna silvestre de la porción terminal de la Cuenca del Plata. Consideraciones biogeográficas y ecológicas. En: J.M. Borthagaray (Ed.). El Río de la Plata como territorio. Ediciones FADU, FURBAN e Infinito, Buenos Aires, Argentina. pp. 99-124.

Simmons, J.E. \& Y. Muñoz-Saba. 2005. Cuidado, manejo y conservación de las colecciones biológicas. Conservation International. Serie Manuales de campo. Bogotá, D.C. Colombia, 288 pp.

Thomas, D. 1906.Holochiluschacarius.AnnualMagazine of Natural History Ser. 7 Vol. 18: 446.

Udrizar Sauthier, W.O., A.M. Abba \& D.E. Udrizar Sauthier. 2010. Nests of Oligoryzomys sp. and Holochilus brasiliensis (Rodentia, Cricetidae) in Eastern Entre Ríos Province, Argentina. Mastozoología Neotropical 17: 207-211.

Valencia, D. 1988. Aves y roedores plaga del cultivo de arroz. El arroz colombiano: retrospectiva, desarrollo, proyección, alimentos, subproductos para ganadería. CO-BAC, Santa Fe de Bogotá, Colombia, pp. 87-89.

Voglino, D., U.F.J. Pardiñas \& P. Teta. 2004. Holochilus chacarius chacarius (Rodentia, Cricetidae) en la provincia de Buenos Aires, Argentina. Mastozoología Neotropical 11: 243-247. 
